\title{
Laccase-catalyzed grafting of lauryl gallate on chitosan to improve its antioxidant and hydrophobic properties
}

$\mathrm{Na}$ Liu ${ }^{*, \dagger, \&}$, Shuzhen $\mathrm{Ni}^{\dagger, \&}$, Hailong Gao ${ }^{\dagger}$, Yongjie Chang ${ }^{\dagger}$, Yingjuan $\mathrm{Fu}^{\dagger}$, Wenxia $\mathrm{Liu}^{\dagger}$, Menghua Qin ${ }^{\S}$

$\dagger$ State Key Laboratory of Biobased Material and Green Papermaking, Qilu University of Technology, Shandong Academy of Sciences, Jinan 250353, China

* Marine Biotechnology, Shandong Industrial Technician College, Weifang 261053, China

$\S$ Organic Chemistry Laboratory, Taishan University, Tai'an 271021, Shandong, China

* Corresponding author: liuna@qlu.edu.cn (Na Liu)

${ }^{\&} \mathrm{Na}$ Liu and Shuzhen Ni contributed equally as the joint first authors. 
Tab.S1 The weight-average, number-average and z-average molecular weight of chitosan samples

\begin{tabular}{llll}
\hline Samples & Native CTS & LG/CTS & Lac/LG-CTS \\
\hline $\mathrm{Mn}$ & $5.249 \mathrm{e}+5(0.8 \%)$ & $5.515 \mathrm{e}+5(1 \%)$ & $5.966 \mathrm{e}+5(0.9 \%)$ \\
$\mathrm{Mw}$ & $5.257 \mathrm{e}+5(0.8 \%)$ & $5.522 \mathrm{e}+5(1 \%)$ & $5.974 \mathrm{e}+5(0.8 \%)$ \\
$\mathrm{Mz}$ & $5.264 \mathrm{e}+5(2 \%)$ & $5.528 \mathrm{e}+5(2 \%)$ & $5.981 \mathrm{e}+5(2 \%)$ \\
$\mathrm{Mw} / \mathrm{Mn}$ & $1.001524(1 \%)$ & 1.001269 & 1.001341 \\
$\mathrm{Mz} / \mathrm{Mn}$ & 1.002857 & 1.002357 & 1.002514 \\
\hline
\end{tabular}

